\title{
Structural Thalamofrontal Hypoconnectivity Is Related to Oculomotor Corollary Discharge Dysfunction in Schizophrenia
}

\author{
Beier Yao, ${ }^{1}$ Sebastiaan F.W. Neggers, ${ }^{2}{ }^{\oplus}$ Martin Rolfs, ${ }^{3}{ }^{\oplus}$ Lara Rösler, ${ }^{2,4}$ Ilse A. Thompson,,${ }^{2,5}{ }^{\circ}$ Helene J. Hopman, ${ }^{2}$ \\ Livon Ghermezi, ${ }^{1}$ René S. Kahn, ${ }^{2,6}$ and ${ }^{\circ}$ Katharine N. Thakkar ${ }^{1,7}$ \\ ${ }^{1}$ Department of Psychology, Michigan State University, East Lansing, Michigan 48824, ${ }^{2}$ Department of Psychiatry, University Medical Center, 3584 CX, \\ Utrecht, The Netherlands, ${ }^{3}$ Department of Psychology and Bernstein Center for Computational Neuroscience, Humboldt-Universität zu Berlin, 10115 \\ Berlin, Germany, ${ }^{4}$ Department of Psychology, Julius Maximilian University of Würzburg, 97070 Würzburg, Germany, ${ }^{5}$ Department of Biomedical Sciences \\ of Cells \& Systems, Section of Cognitive Neurosciences, University of Groningen, University Medical Center Groningen, 9713 GZ, Groningen, The \\ Netherlands, ${ }^{6}$ Department of Psychiatry, Icahn School of Medicine at Mount Sinai, New York, New York 10029, and ${ }^{7}$ Division of Psychiatry and Behavioral \\ Medicine, Michigan State University, Grand Rapids, Michigan 49503
}

By predicting sensory consequences of actions, humans can distinguish self-generated sensory inputs from those that are elicited externally. This is one mechanism by which we achieve a subjective sense of agency over our actions. Corollary discharge (CD) signals"copies" of motor signals sent to sensory areas-permit such predictions, and CD abnormalities are a hypothesized mechanism for the agency disruptions in schizophrenia that characterize a subset of symptoms. Indeed, behavioral evidence of altered CD, including in the oculomotor system, has been observed in schizophrenia patients. A pathway projecting from the superior colliculus to the frontal eye fields (FEFs) via the mediodorsal thalamus (MD) conveys oculomotor CD associated with saccadic eye movements in nonhuman primates. This animal work provides a promising translational framework in which to investigate $\mathrm{CD}$ abnormalities in clinical populations. In the current study, we examined whether structural connectivity of this MD-FEF pathway relates to oculomotor CD functioning in schizophrenia. Twenty-two schizophrenia patients and 24 healthy control participants of both sexes underwent diffusion tensor imaging, and a large subset performed a trans-saccadic perceptual task that yields measures of $\mathrm{CD}$. Using probabilistic tractography, we identified anatomical connections between FEF and MD and extracted indices of microstructural integrity. Patients exhibited compromised microstructural integrity in the MD-FEF pathway, which was correlated with greater oculomotor CD abnormalities and more severe psychotic symptoms. These data reinforce the role of the MD-FEF pathway in transmitting oculomotor CD signals and suggest that disturbances in this pathway may relate to psychotic symptom manifestation in patients.

Key words: corollary discharge; DTI; eye movements; predictive coding; probabilistic tractography; psychosis

Significance Statement

People with schizophrenia sometimes experience abnormalities in a sense of agency, which may stem from abnormal sensory predictions about their own actions. Consistent with this notion, the current study found reduced structural connectivity in patients with schizophrenia in a specific brain pathway found to transmit such sensorimotor prediction signals in nonhuman primates. Reduced structural connectivity was correlated with behavioral evidence for impaired sensorimotor predictions and psychotic symptoms.

\section{Introduction}

Agency disturbance is a defining feature of schizophrenia. It is obvious in passivity delusions - the belief that one is controlled

Received June 9, 2018; revised Nov. 30, 2018; accepted Jan. 2, 2019.

Author contributions: S.F.W.N., M.R., R.S.K., and K.N.T. designed research; L.R., I.A.T., H.J.H., and K.N.T. performed research; B.Y., S.F.W.N., L.R., L.G., and K.N.T. analyzed data; B.Y. wrote the paper.

This work was supported in part by National Institutes of Health Grant R01-MH-112644 (K.N.T.), National Institute of Mental Health Grant R21-MH-115297-01 (K.N.T.), a NARSAD Young Investigator Award from the Brain and Behavior Foundation (K.N.T.), a Netherlands Organization for Scientific Research Rubicon grant (K.N.T.), a short-stay fellowship from Utrecht University (K.N.T.), and a University of Utrecht Neuroscience and Cognition grant (S.F.W.N.). by outside forces_-but may also underlie psychotic symptoms broadly (Feinberg and Guazzelli, 1999). A sensorimotor account of agency disturbances posits a key role of altered corollary discharge (CD) signals_ “copies" of motor signals sent to sensory

Hammers adult maximum probability atlas @ Copyright Imperial College of Science, Technology and Medicine 2007. All rights reserved.

The authors declare no competing financial interests.

Correspondence should be addressed to Katharine N. Thakkar at kthakkar@msu.edu.

https://doi.org/10.1523/JNEUROSCI.1473-18.2019

Copyright $\odot 2019$ the authors $\quad 0270-6474 / 19 / 392102-12 \$ 15.00 / 0$ 
A

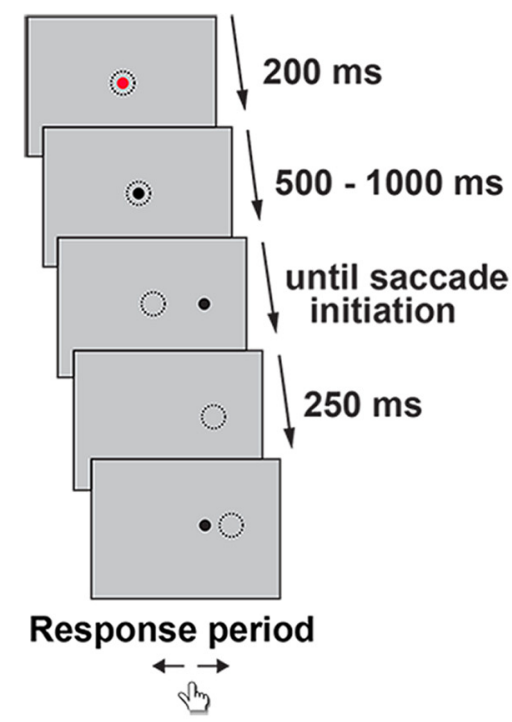

B
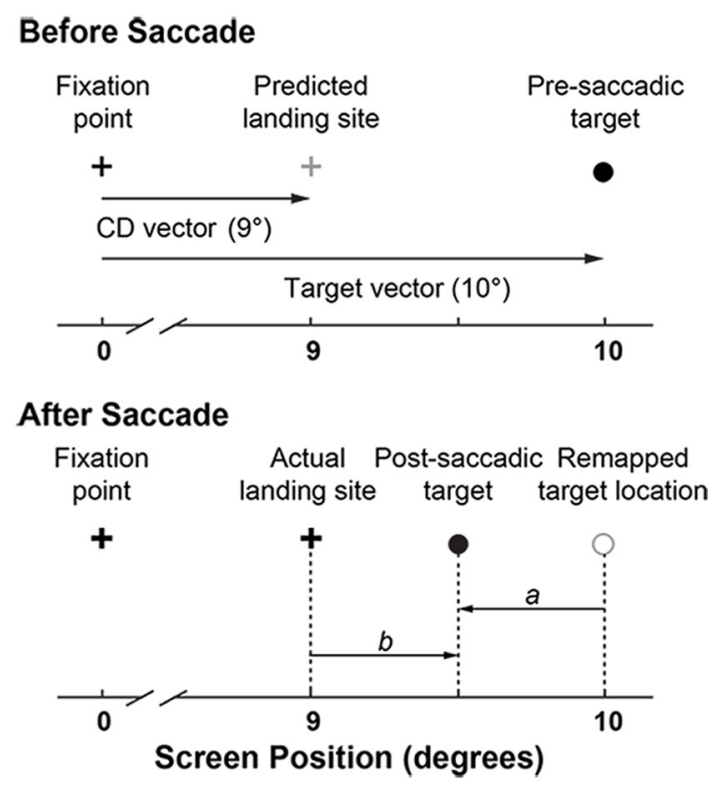

Figure 1. A, Blanking task. Dotted circles represent gaze positions. B, Perceptual judgements in the blanking task. Top, After the stimulus appears at the presaccadic location (black dot), the motor command to execute a saccade toward this location is generated and a $C D$ vector associated with this motor command can be computed. In this example, the predicted saccade landing site (gray cross) based on the CD signal associated with the saccade motor command will fall short of the target. Bottom, Upon saccade initiation, the stimulus will disappear and reappear (black target) to the left or right of the presaccadic location (empty dot). If the participant can use CD to represent the presaccadic location correctly relative to the new gaze position, then vector $a$ should match the actual stimulus displacement. In this example, the postsaccadic target location is to the left of the presaccadic location, so the participant should judge the displacement as backward. However, if the participant does not have access to complete CD information, saccade landing site may be used as a proxy of the presaccadic location. In this case, the participant will perceive vector $b$ as the target displacement and indicate a forward response instead. See Materials and Methods section for more task details.

areas to predict the impending sensory inputs (Feinberg, 1978). A mismatch between predicted and actual sensory inputs arguably leads to misattributing self-generated actions to external forces.

There is strong behavioral evidence supporting the involvement of $\mathrm{CD}$ in the generation of eye movements, and primate neurophysiology studies have provided direct evidence for a pathway from motor neurons in the superior colliculus to sensory neurons in the frontal eye fields (FEFs) via the mediodorsal thalamus (MD) that conveys CD signals (Sommer and Wurtz, 2008). Consequently, the oculomotor system becomes an ideal framework in which to develop a mechanistic understanding of the faulty sensorimotor predictions that may underlie agency disturbances. Indeed, there is a considerable body of behavioral evidence suggesting oculomotor $\mathrm{CD}$ abnormalities in psychosis (for reviews see Thakkar et al., 2017; Bansal et al., 2018b). Furthermore, there is growing evidence for thalamocortical dysconnectivity in schizophrenia that relates to symptomatology and functioning (for reviews see Pergola et al., 2015; Canu et al., 2015), of which altered CD signaling may be a downstream consequence. In this study, we relate, for the first time, measures of oculomotor CD and thalamocortical connectivity in schizophrenia patients.

One important function served by $\mathrm{CD}$ signals in oculomotor processes is to maintain the perception of a continuous world despite eye movements causing displacement of images across the retina (Cavanagh et al., 2010). One commonly used task to assess this so-called visual stability is the blanking task (Fig. 1A; Deubel et al., 1996), where a target disappears briefly upon saccade initiation and reappears at a displaced location upon landing. Participants indicate the perceived direction of the displacement relative to its original (presaccadic) location. Because saccadic eye movements are often imprecise, participants cannot rely on the position of gaze following the eye movement (saccade landing site) to inform them of the presaccadic target location and the displacement direction. Indeed, in healthy observers, perceptual judgments are independent of where the saccade landed, suggesting that observers did not use saccade landing site as a proxy for the presaccadic target location (Collins et al., 2009). Rather, they appeared to use a CD signal associated with the saccade to correctly represent the presaccadic target location and localize the postsaccadic target. If CD signals are compromised, however, participants may not have information on the spatial accuracy of their saccade and can only use the saccade landing site to make the judgment. Accurate performance on this task, therefore, requires an intact $\mathrm{CD}$ signal (Fig. 1B). We and others recently found impaired performance in the blanking task in patients with schizophrenia, who showed greater reliance on saccade landing site than the presaccadic location when making perceptual judgements (Rösler et al., 2015; Bansal et al., 2018a). Such reliance was bigger in patients with more severe positive symptoms, supporting the theory that abnormal CD is a key mechanism of psychosis (Feinberg and Guazzelli, 1999). The blanking task has also been used in primate studies (Joiner et al., 2013). Consistent with the posited role of MD in relaying oculomotor CD signals, performance is disturbed by MD inactivation (Cavanaugh et al., 2016).

The current study used diffusion tensor imaging (DTI) to explore the relationships among oculomotor $\mathrm{CD}$, psychotic symptoms, and structural integrity of a probabilistically defined MD-FEF pathway. We expected reduced structural connectivity in this pathway in patients with schizophrenia relative to healthy control participants which would relate to CD signal integrity and psychotic symptom severity. Results from this study will add to our understanding of both the neural pathways involved in oculomotor $\mathrm{CD}$ signaling in the human brain and their putative involvement in the self-related symptoms of schizophrenia. 
Table 1. Demographic information (see Table 1-1, available at https://doi.org/ 10.1523/JNEUROSCI.1473-18.2019.t1-1, for the demographic information of the smaller subsample with both DTI data and usable data on the blanking task)

\begin{tabular}{lcclr}
\hline & $\begin{array}{l}\text { Healthy control } \\
\text { participants } \\
(n=24)\end{array}$ & $\begin{array}{l}\text { Patients with } \\
\text { schizophrenia } \\
(n=22)\end{array}$ & Statistic & $p$ value \\
\hline Age (years) & $34.9(8.1)$ & $37.4(7.8)$ & $t=-1.1$ & 0.29 \\
Sex (female/male) & $11 / 13$ & $5 / 17$ & $\chi^{2}=2.7$ & 0.13 \\
IO $^{a}$ & $100.4(13.8)$ & $96.0(12.8)$ & $t=1.1$ & 0.29 \\
Education $^{b}$ & $7.2(1.3)$ & $4.8(1.7)$ & $t=5.2$ & $<0.001$ \\
Handednessc $^{c}$ & $0.89(0.41)$ & $0.85(0.45)$ & $t=0.35$ & 0.73 \\
IIIness duration (years) & & $14.2(5.2)$ & & \\
PANSS positive & & $11.6(5.2)$ & & \\
PANSS negative & & $13.1(6.3)$ & & \\
PANSS general & & $25.0(7.9)$ & & \\
CPZ equivalent (mg) & & $269.6(247.9)$ & & \\
\hline
\end{tabular}

Values are reported as mean (SD), unless otherwise indicated.

${ }^{a}$ Based on the Nederlandse Leestest voor Volwassenen.

${ }^{b}$ Education category: $0=<6$ years of primary education; $1=$ finished 6 years of primary education; $2=6$ years of primary education and low-level secondary education; $3=4$ years of low-level secondary education; $4=4$ years of average-level secondary education; $5=5$ years of average-level secondary education; $6=4$ years of secondary vocational training; $7=4$ years of high-level professional education; $8=$ university degree.

'Based on the Edinburgh Handedness Inventory; scores range from 0 indicating complete left-handedness to 1 indicating complete right-handedness.

\section{Materials and Methods}

\section{Overview}

Individuals with schizophrenia and healthy control participants underwent DTI; a large subset also performed the blanking task during a separate session. Detailed results of blanking task performance were reported in the study by Rösler et al. (2015). Putative white matter tracts connecting the FEFs and MD were computed using probabilistic tractography (Behrens et al., 2003b, 2007). Mean microstructural integrity measures were calculated for each participant. These indices of microstructural integrity of white matter tracts were used for further group comparisons and correlation analyses with behavioral indices of CD obtained from the blanking task, as well as symptom scores within the patient group.

\section{Participants}

Twenty-two antipsychotic-medicated patients with schizophrenia of either sex were recruited from a longitudinal study [Genetic Risk and Outcome in Psychosis (GROUP) Investigators, 2011] and an outpatient psychiatric facility in The Netherlands. Schizophrenia or schizoaffective disorder diagnoses were based on Diagnostic and Statistical Manual of Mental Disorders, fourth edition (DSM-IV) criteria and verified with the Comprehensive Assessment of Symptoms and History interview (Andreasen et al., 1992) or Schedules for Clinical Assessment for Neuropsychiatry, version 2.1 (Wing et al., 1990). Chlorpromazine (CPZ) equivalent antipsychotic dosages were calculated for each patient (Woods, 2003). Clinical symptoms were assessed with the Positive and Negative Syndrome Scale (PANSS; Kay et al., 1987). IQ was assessed with the Nederlandse Leestest voor Volwassenen (Mulder et al., 2012). Twenty-four control participants of either sex without a personal history of DSM-IV Axis I diagnosis or family history of schizophrenia spectrum disorders were recruited via community advertisements. Participants were excluded if they had a history of head trauma or neurological illness, or recent substance abuse or dependence. The patients and control participants were matched for sex, age, IQ, and handedness (Table 1, detailed participant characteristics). Nineteen patients and 17 control participants had both DTI data and usable data on the blanking task; this subset was used for correlational analyses between blanking task performance and diffusivity measures. Control participants and patients in this subset also did not differ on sex, age, IQ, and handedness (Table 1-1, available at https://doi.org/10.1523/JNEUROSCI.1473-18.2019.t1-1).

All participants gave written informed consent and were reimbursed for participation. The study was approved by the Human Ethics Committee of the University Medical Center, Utrecht, The Netherlands.
DTI

The following gives a broad overview of the DTI acquisition and analysis steps, which are detailed in subsequent sections.

- Image acquisition. All DTI data were acquired on a $3 \mathrm{~T}$ scanner with 30 different gradient directions to allow for computation of principal fiber orientations within the brain.

- Preprocessing. All scan images were corrected for spatial distortions and eddy current distortions. Images were spatially aligned with one another, and then fit to a tensor model to derive the diffusivity measures that served as an index of microstructural integrity. Finally, spatial normalization parameters were obtained, which allowed for the transformation of regions of interest (ROIs) and tractography results between each participant's individual (i.e., native) space and a standard template.

- Regions of interest. FEF was functionally defined, using functional MRI (fMRI) data obtained from the same participants while they made saccades. MD was defined anatomically using a probabilistic atlas. Corpus callosum was included as a control tract and was also anatomically defined using a probabilistic atlas.

- Probabilistic tractography. Putative white matter tracts connecting the FEFs and MD were computed using probabilistic tractography, an analysis technique that reconstructs anatomical pathways (presumably white matter tracts) between brain regions based on a distribution profile of probable fiber orientations in each voxel (Behrens et al., 2003b, 2007). Participants' individual probabilistic tracts were transformed into a standard space, and the results were averaged within each group. Then, a statistical threshold was applied to these group-averaged tracts. These thresholded tracts were binarized and constituted the final tracts for each group. As part of the tractography analysis, a seed-to-target image was computed for each individual, indicating the level of connectivity to FEFs within each voxel of MD. These images were normalized and averaged to locate the part of MD that has the highest connectivity with FEFs.

- Diffusivity measures of microstructural integrity. Within each groupaveraged MD-FEF tract as well as the control corpus callosum tract, mean diffusivity measures were calculated for each participant. These diffusivity measures served as indices of microstructural integrity. Multiple measures of diffusivity within each tract were calculated because these different measures have different physiological correlates and thus provide slightly different information about structural integrity.

Image acquisition. All DTI data were acquired at the University Medical Center Utrecht on a an Achieva 3 T scanner (Philips Medical Systems) equipped with an eight-channel head coil allowing parallel imaging. Two diffusion images were acquired using single-shot echoplanar imaging sequences, consisting of 30 diffusion-weighted scans $\left(b=1000 \mathrm{~s} / \mathrm{mm}^{2}\right)$ with noncollinear gradient directions and one image without diffusion weighting $\left(b=0 \mathrm{~s} / \mathrm{mm}^{2}\right)$, covering the entire brain [repetition time $(\mathrm{TR})=7057 \mathrm{~ms}$; echo time $(\mathrm{TE})=68 \mathrm{~ms}$; field of view $=240 \times 240 \times$ $150 \mathrm{~mm}$; in-plane resolution $=1.875 \times 1.875 \mathrm{~mm}$; slice thickness $=2$ $\mathrm{mm}$; no slice gap; 75 axial slices; matrix size, $128 \times 99 \mathrm{~mm}]$. The diffusion-weighted scans were measured twice, once with phaseencoding direction reversed (first scan, posterior-anterior; second scan, anterior-posterior), to correct for susceptibility-induced spatial distortions (Andersson and Skare, 2002). For registration purposes, a wholebrain three-dimensional T1-weighted scan $(200$ slices; TR $=10 \mathrm{~ms}$; $\mathrm{TE}=4.6 \mathrm{~ms}$; flip angle $=8^{\circ}$; field of view, $240 \times 240 \times 160 \mathrm{~mm}$; voxel size: $0.75 \times 0.8 \times 0.75 \mathrm{~mm}$ ) was acquired.

Preprocessing. The diffusion-weighted scans were preprocessed and analyzed using FSL 5.0 (FMRIB Software Library; www.fmrib.ox.ac.uk/ fsl). As DTI scans suffer from spatial distortions along the phaseencoding direction, two diffusion-weighted scans were acquired with reversed phase-encoding blips, resulting in pairs of images with distortions going in opposite directions. From these two images, the offresonance field was estimated using a method similar to that described by Andersson and Skare (2002) as implemented in FSL (Smith et al., 2004). Next, the 30 diffusion-weighted images from each phase-encoding direction were realigned to the $b 0$ image using affine registration, and eddy current correction was applied. The eddy-corrected scans with opposite phase-encoding blips were then combined into a single corrected image using the previously estimated off-resonance field. A brain mask was 

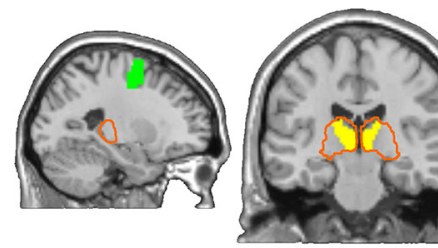

$\mathrm{X}: 34$

Y: 53
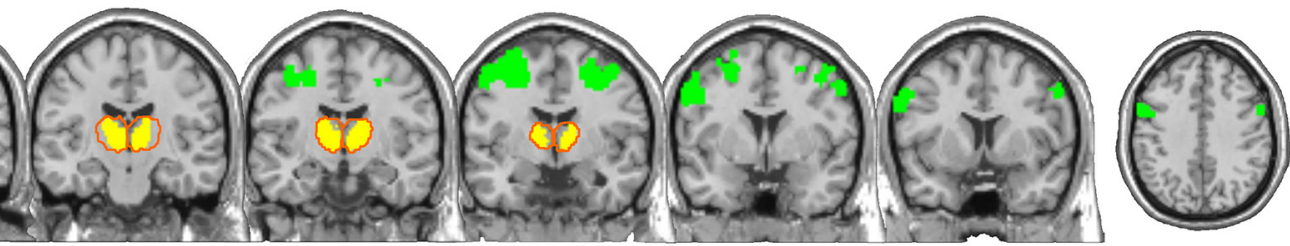

56

59

62

65

68

Z: 56
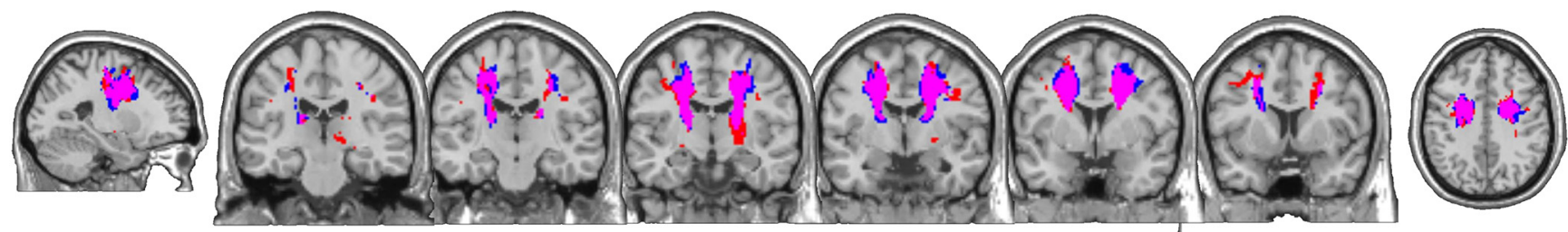

Figure 2. Visualization of the probabilistic tractography results of the mediodorsal thalamus-frontal eye fields pathway in patients and control participants. Top, ROI masks displayed on a standard-space brain in coronal view on multiple slices from anterior to posterior: FEF, Green; MD, yellow. As a reference point, the outline of thalamus as defined by the Harvard-0xford thalamic connectivity atlas is displayed in orange (thresholded at $\geq 25 \%$ of connectivity to anywhere in the cortex). Bottom, MD-FEF white matter pathways averaged and thresholded within each group, displayed on a standard-space brain in coronal view on multiple slices from anterior to posterior: control participants' tracts, red; patients' tracts, blue; overlapping areas, pink. Coordinates of each slice in standard (MNI) space are presented in the middle.

created for the mean b0 image and applied to all diffusion-weighted images.

Diffusion data were then fit to a tensor model using the DTIFIT command in the FMRIB library, resulting in the calculation of three principal eigenvalues $\left(\lambda_{1}, \lambda_{2}, \lambda_{3}\right)$ at each voxel. These eigenvalues were used to calculate, at each voxel, the following four diffusivity measures of interest: fractional anisotropy (FA), mean diffusivity, radial diffusivity (RD), and axial diffusivity (AD). FA is scaled from 0 (completely isotropic) to 1 (completely anisotropic) and is calculated using the following formula (Basser and Pierpaoli, 1996):

$$
F A=\sqrt{\frac{1}{2}} \frac{\sqrt{\left(\lambda_{1}-\lambda_{2}\right)^{2}+\left(\lambda_{2}-\lambda_{3}\right)^{2}+\left(\lambda_{3}-\lambda_{1}\right)^{2}}}{\sqrt{\lambda_{1}^{2}+\lambda_{2}^{2}+\lambda_{3}^{2}}} .
$$

Mean diffusivity is the average of the eigenvalues: mean diffusivity $=$ $\left(\lambda_{1}+\lambda_{2}+\lambda_{3}\right) / 3$. Axial diffusivity refers to the amplitude of the eigenvalue along the principal (axial) tensor direction $\left(\mathrm{AD}=\lambda_{1}\right)$, while radial diffusivity refers to the average amplitude of the eigenvalues perpendicular to the principal tensor direction $\left[\mathrm{RD}=\left(\lambda_{2}+\lambda_{3}\right) / 2\right]$.

DTI analyses must be performed in native space as diffusion gradients are specified in this space. Thus, as a final step, we performed spatial normalization in order for ROIs to be transformed from a standard [Montreal Neurological Institute (MNI)] space into each individual's native space, and for probabilistic tracts in native space to be compared across individuals. Accordingly, each subject's anatomical T1-weighted volume was realigned to their mean $b 0$-weighted image, and subsequently segmented into gray matter, white matter, and CSF, and normalized to MNI space using the unified segmentation algorithm as implemented in SPM8 (Ashburner and Friston, 2005). Individual white matter masks were created for each subject based on the segmentation procedure described above, which creates tissue probability maps for gray matter, white matter, and CSF. Binary white matter masks comprised those voxels that had a higher probability of being identified as white matter than as gray matter or CSF.

Regions of interest. We included two ROIs in each hemisphere: FEF and MD (Fig. 2, top, illustration of the FEF and MD masks). Because FEF does not have clear anatomical boundaries, it was defined based on group-level fMRI maps derived from the same participants performing a different saccadic eye movement task in the scanner (Thakkar et al., 2014) during the same session as the DTI data were acquired. More specifically, FEFs were created based on areas that showed greater activation on trials during which participants were making saccades versus where they were fixating (threshold, $p<0.001$ uncorrected), including both medial and lateral parts of FEFs (Neggers et al., 2012). The thalamus was localized using the Harvard-Oxford thalamic connectivity atlas, which is a probabilistic atlas of seven subthalamic regions segmented according to their white matter connectivity to cortical areas (Behrens et al., 2003a). Within these seven thalamic subdivisions, each voxel represents the proportion of individuals for whom that voxel had a probability of at least 0.25 of being connected to the relevant cortical region. MD was operationally defined as the region of the thalamus at which at least $25 \%$ of individuals showed a probability of at least 0.25 with the prefrontal cortex. Using the $25 \%$ threshold has been shown to result in thalamic subdivisions that map well onto findings from cytoarchitectonic data (Johansen-Berg et al., 2005). To examine whether the tract-behavior correlations were unique to MD-FEF pathway, we also included corpus callosum as a control ROI, localized using the Hammers adult maximum probability atlas (Hammers et al., 2003; thresholded at 3; www. brain-development.org). All ROIs (i.e., FEFs, MD, and corpus callosum) were defined in MNI space and transformed into each individual's native space using the inverse warping parameters defined during spatial normalization. They were then masked by white matter, defined individually as described in the previous section.

Probabilistic tractography. We performed probabilistic tractography between $\mathrm{MD}$ and FEF in both hemispheres from both directions (i.e., $\mathrm{MD}$ to FEF and FEF to MD), each serving as both a seed (start point) and a target (end point), since diffusion MRI cannot distinguish between afferent and efferent axonal fibers. The distribution profile of probabilistic connectivity was computed by iteratively sending out 5000 streamlines from the seed area, going in a direction drawn from a distribution around the principal diffusion direction voxel by voxel, until it was determined to be structurally impossible for a white matter tract to continue. Only streamlines that reached the target successfully were included in the analysis, and streamlines were not allowed to continue after reaching the target. Two crossing fibers per voxel were allowed. In addition, the mid-sagittal plane was used as an exclusion mask to avoid streamlines traveling falsely into the other hemisphere (Mori and van Zijl, 2002; Mori and Zhang, 2006; Landman et al., 2012). Upon visual examination of preliminary tractography results, an axial plane located one voxel beneath MD was added as another exclusion mask to avoid streamlines traveling in the opposite direction of FEF and looping back on themselves. Final tractography results for each individual were visually inspected. We were able to successfully construct a pathway between MD and FEF in both hemispheres for all participants.

The end result of the probabilistic tractography analysis is a map with a "connectivity value," which indicates the number of streamlines passing through that specific voxel, at each voxel. Higher values indicate a higher probability that the voxel belongs to that particular tract, specified on the basis of seed and target regions. To perform group analyses, indi- 
vidual tractography results were transformed to MNI space. To make sure that only white matter structures were included in the analyses, each individual's whole-brain white matter map was used as a mask to preserve connectivity values in these structures only. After that, these values were averaged across participants within each group.

Connectivity values are determined not only by actual structural connectivity, but are also influenced by other factors like size of the seed ROI (i.e., bigger seeds send out more streamlines). Thus, higher connectivity values in a given tract does not necessarily indicate a larger probability of an actual structural connection, and the number of streamlines passing through a given voxel cannot be directly compared across seed/target combinations. Therefore, to avoid potential biases, we did not use the same absolute value for thresholding each group-averaged tract but used instead the top $0.27 \%$ ( 3 SDs above the mean in normal distribution) of all voxels as the threshold. After applying the threshold, these group tracts were binarized into masks per tract. Masks from both directions (i.e., FEF to MD and MD to FEF) were considered to be equally accurate and were combined to achieve a single mask for the MD-FEF pathway per each group. These normalized, group-averaged, and binarized tracts will be henceforth referred to as the MD-FEF tracts.

As a follow-up analysis to examine which part of the MD has the highest connectivity with FEF, we normalized each individual's seed-totarget image and averaged them across all participants. In seed-to-target images, each voxel of the seed region (in this case, MD) denotes the number of streamlines initiated from that voxel that reached the target region (in this case, FEF). Distance correction was used to correct for the loss of connectivity when the distance from the seed mask increases.

Diffusivity measures of microstructural integrity. Fractional anisotropy is the most widely used measure of the microstructural integrity of white matter. Higher fractional anisotropy values mean higher anisotropy and lower diffusivity (e.g., better microstructural integrity and consequently more efficient connectivity). However, fractional anisotropy can be difficult to interpret in isolation. A decrease in fractional anisotropy could be caused by multiple factors, such as white matter neuropathology, fiber crossing, and normal aging (Alexander et al., 2007). Therefore, it is recommended to use multiple diffusivity measures (e.g., mean diffusivity, axial diffusivity, and radial diffusivity) for better understanding of the microstructure of white matter. Higher values for mean diffusivity mean more diffusivity (e.g., less efficient connectivity), and higher values for axial diffusivity and radial diffusivity mean higher diffusivity along specific directions (i.e., along the principal fiber direction and perpendicular to the principal direction, respectively). Radial diffusivity appears to be a more sensitive indicator of myelination, while reduced axial diffusivity indicates axonal degeneration (Song et al., 2002). Mean diffusivity has been found to be most sensitive to white matter maturation and aging (Abe et al., 2002; Snook et al., 2007).

For each participant, diffusivity measures of microstructural integrity of brain fibers (fractional anisotropy, radial diffusivity, mean diffusivity, and axial diffusivity) were extracted and averaged within both the left and right MD-FEF tracts, as well as in the corpus callosum control tract in each subject's native space.

\section{Blanking task}

Apparatus and stimuli. Participants sat in a dimly lit room with their head stabilized $68 \mathrm{~cm}$ in front of a 24 inch computer screen (spatial resolution, $1920 \times 1080$ pixels; vertical refresh rate, $100 \mathrm{~Hz}$ ). Stimuli were black and red dots with a diameter of $0.2^{\circ}$ presented on a gray background. An EyeLink 1000 (SR Research) was used to track eye position. Manual responses were recorded using a button box. Stimulus presentation and response collection were operated through MATLAB (MathWorks) with the Psychophysics (Brainard, 1997) and Eyelink (Cornelissen et al., 2002) toolboxes.

Design and procedure. The blanking task putatively indexes the degree to which CD influences visual perception immediately following a saccade (Fig. 1A). Each trial started with the participant fixating on a red circle. To reduce anticipation effects or stereotypical behavior (Collins et al., 2009), the circle randomly appeared at one of six locations (a combination of $\mathrm{a}-1^{\circ}$ or $1^{\circ}$ of visual angle displacement horizontally and $\mathrm{a}-1^{\circ}$, $0^{\circ}$, or $1^{\circ}$ displacement vertically relative to the center of the screen) with

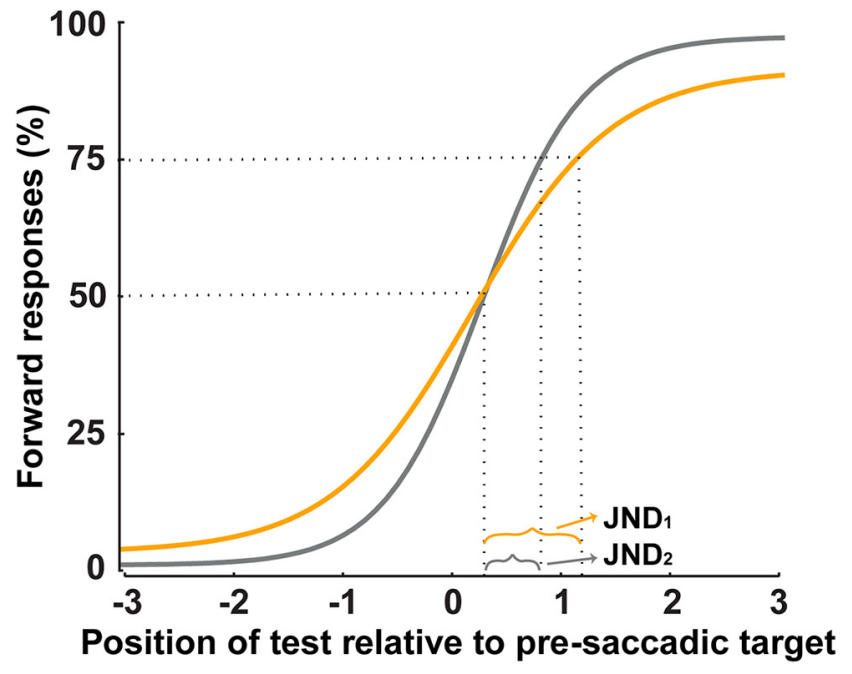

Figure 3. Illustration of JND calculation. JND was derived from the psychometric curve plotting the proportion of forward responses as a function of target displacement and calculated as the difference in target displacements between the $50 \%$ and $75 \%$ points on the curve. On the $x$-axis, negative values mean backward jumps (toward the fixation) and positive values mean forward jumps (away from the fixation) relative to the presaccadic location. Larger JND values are indicative of a flatter slope of this function, suggesting reduced sensitivity to the actual target displacement.

equal probability. Once the eye tracker detected that the participant had maintained fixation for $200 \mathrm{~ms}$, the circle turned black. After a random delay of 500-1000 ms, the circle disappeared and reappeared at a new location $10^{\circ}$ to the left or right of the fixation position (presaccadic location). The participant was instructed to look at the stimulus as quickly as possible. Once the participant initiated a saccade, the stimulus would disappear (i.e., blank) for $250 \mathrm{~ms}$ and reappear at a location (postsaccadic location) somewhere between $-3^{\circ}$ and $3^{\circ}$ (in increments of $0.5^{\circ}$ ) to the left or right of the presaccadic location and then stay on the screen for the remainder of the trial. The participant was then asked to report in which direction the stimulus jumped relative to its presaccadic location by key pressing. Although participants indicated a left or right response, for analysis purposes, we recoded these responses, as follows: "forward" means jumping away from the initial fixation position; and "backward" means jumping toward the initial fixation. The combination of 6 fixation positions $\times 13$ postsaccadic displacements $\times 2$ directions (left, right) resulted in 156 total trials. The duration of the task varied across participants and typically ranged from 15 to $30 \mathrm{~min}$. A boundary technique was used for on-line saccade detection. Saccade initiation was defined as eyes moving beyond a $2^{\circ}$ window around the fixation dot. Saccades detected before target onset would trigger a warning message on the screen and a restart of the trial.

Data analysis. Based on the results reported in the study by Rösler et al. (2015), which are summarized in the Results section, two measures of $\mathrm{CD}$ were used to relate to FEF-MD structural connectivity, as follows: the just noticeable difference (JND) and the loss slope. The following describes how we derived each of these measures.

To obtain the JND for each individual, trials were collapsed across fixation positions and saccade directions. We then fit a logistic function to the data, plotting the percentage of forward responses as a function of target displacement (i.e., the position of postsaccadic location relative to the presaccadic location; Fig. 3, examples). We derived the JND from this psychometric curve as the difference in target displacements between the points where the function reached $50 \%$ and $75 \%$ of its full growth. Because we did not find any effect of saccade direction on JND in our previous study (Rösler et al., 2015), we calculated JND using the psychometric function that collapsed across the two saccade directions. This measure indicates individuals' sensitivity to target displacement. We expect that, with the loss of CD signals, participants would rely less on the actual target displacements and more on the landing site of their gaze. 


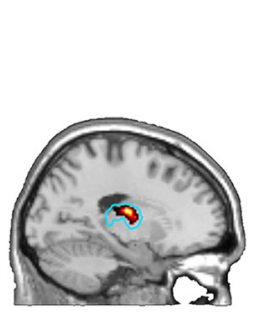

$\mathrm{X}: 37$

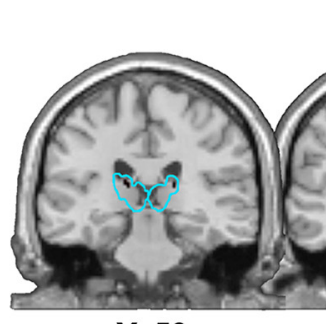

Y: $\mathbf{5 0}$ $\overline{15}$ 15

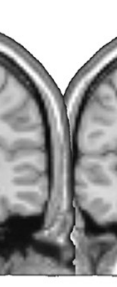

53

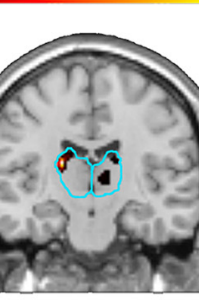

56

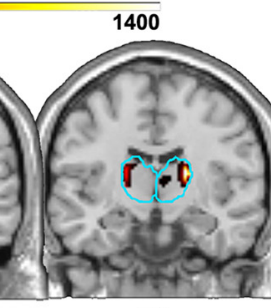

59

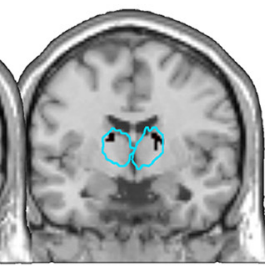

62

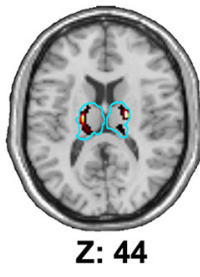

Z: 44

Figure 4. Connectivity profile of the tract from MD to FEF. The heatmap represents the areas of MD that show the strongest connectivity with the FEF. Brighter regions of the MD indicate higher connectivity with FEF. These heatmaps are constructed by calculating, for each participant, the number of streamlines from each voxel in the mediodorsal thalamus (outlined in cyan) that arrived at the FEF in each individual. These individual heatmaps were spatially normalized and averaged across participants and are shown here on a standard-space (MNI) brain. Numbers at the end of the color scale refer to the number of streamlines initiating from a single voxel within MD that reached the FEF.

Accordingly, they would be less sensitive to actual target displacements, which would manifest in a larger JND.

To calculate the loss slope, trials were collapsed across target displacements for each individual. To maximize the power given the relatively small number of trials, we collapsed across leftward and rightward saccades. We then derived landing site errors (i.e., distance between the actual saccade landing site and the presaccadic location) for each bin between $-8^{\circ}$ and $8^{\circ}$ in $0.5^{\circ}$ intervals. For each bin, we calculated the mean saccade landing site. The loss slope was derived from a weighted linear regression where the percentage of forward responses corresponding to each bin was fit against the mean saccade landing site per bin, weighted by the number of trials per bin. Because individuals with intact CD signals should not rely on saccade landing sites to make perceptual judgments about target displacements, the slope should be almost zero in control participants (Collins et al., 2009; Ostendorf et al., 2010). More negative slope values indicate a stronger reliance on the landing site and therefore a larger loss of CD signals, hence the term "loss slope."

\section{Statistical analyses}

First, we analyzed group differences in tract locations using SPM8 (http://www.fil.ion.ucl.ac.uk/spm/software/spm8). For each subject, in each hemisphere, a probability value was calculated for each voxel by dividing the number of tracts going through the voxel by the total number of tracts in the MD-FEF pathway (i.e., the number of streamlines sent out from the seed structure that made it to the target structure) after combining results from both directions (Marenco et al., 2012). This value indicates the probability of the specific voxel belonging to the MDFEF structural connection. Then between-group $t$ tests were performed on probability values at each voxel on the whole-brain level. Results were examined at $p<0.05$, using familywise error correction to account for multiple comparisons.

Next, we performed group comparisons of diffusivity measures and related them to symptoms and performance using SPSS Statistics version 20.0 (IBM). To compare the microstructural integrity of MD-FEF white matter tracts, we conducted repeated-measures ANOVAs on each of the four microstructural integrity measures (fractional anisotropy, mean diffusivity, radial diffusivity, and axial diffusivity), including diagnostic group as a between-subjects variable. Because the hemispheric effects on anatomical thalamocortical connectivity are unclear yet important to our understanding of disease mechanisms (Ramsay and MacDonald, 2018), we also included hemisphere as a within-subject variable.

Since we were interested in the specific influence of structural connections between MD and FEF (instead of global white matter integrity) on behavior, mean integrity measures over the whole brain were regressed out of the tract measures that did not differ between hemispheres (Kelly et al., 2018). For measures with a significant hemisphere effect or hemisphere $\times$ group interaction, mean integrity measures over each hemisphere were regressed out of the corresponding tract measures. These standardized residuals (within each hemisphere or collapsed across hemispheres when there was no hemisphere difference) were used for all of the correlation analyses. All standardized values are 0 when equal to the whole-brain average of the corresponding measure. For standardized residuals of fractional anisotropy, higher values indicate more anisotro- pic voxels (i.e., higher structural integrity), and lower values indicate more isotropic voxels (i.e., reduced structural integrity). For standardized residuals of mean diffusivity, radial diffusivity, and axial diffusivity, higher values indicate higher diffusivity. We performed Pearson correlation analyses between two behavioral measures (loss slope and JND) and the standardized residuals of diffusivity measures. Using patients' data only, we calculated Spearman's rank correlation coefficients $\left(r_{s}\right)$ between PANSS (Kay et al., 1987) subscale scores (positive and negative symptoms) and standardized residuals of diffusivity measures. To examine the potential confounding effects of antipsychotic use, the standardized (chlorpromazine equivalent) medication dose was correlated with behavioral measures and microstructural integrity measures using Spearman's rank correlation.

To examine whether the putative influence of structural connectivity on behavior was unique to the MD-FEF pathway, corpus callosum was included as a control pathway. Mean integrity measures over the whole brain were regressed out of the tract integrity measures of corpus callosum as well. Pearson correlation analyses were conducted between two behavioral measures (loss slope and JND) and the standardized residuals of diffusivity measures extracted from corpus callosum.

\section{Results \\ MD-FEF connectivity}

The spatially normalized, group-averaged, and statistically thresholded MD-FEF probabilistic tracts in both the left and right hemisphere are shown in Figure 2 (bottom). We performed a between-group $t$ test on whole-brain probability values at each voxel. No significant differences were found between the patients and control participants at a familywise error-corrected threshold of $p<0.05$ or at a more liberal uncorrected threshold of $p<$ 0.001 . These results argue against any gross anatomical differences in the location of the MD-FEF pathway between patients and control participants. The follow-up seed-to-target analysis revealed that the strongest connectivity to the FEF was in the most lateral aspects of the MD (Fig. 4).

Next, we conducted four repeated-measures ANOVAs on each of the mean microstructural integrity measures (i.e., fractional anisotropy, mean diffusivity, radial diffusivity, axial diffusivity) extracted from the MD-FEF tracts, using diagnostic group as a between-subjects variable and hemisphere as a withinsubject variable (Fig. 5). Means and SDs are presented in Table 2. Again, higher microstructural integrity (and hence more efficient connectivity) is indicated by higher fractional anisotropy values. Higher diffusivity (and hence lower microstructural integrity in general or along certain direction) is indicated by higher mean diffusivity, radial diffusivity, and axial diffusivity values. For fractional anisotropy, mean diffusivity, and radial diffusivity, there was a significant effect of group, such that patients showed evidence of reduced microstructural integrity of the MD-FEF pathway (fractional anisotropy: $F_{(1,44)}=11.05, p=0.002$, partial 

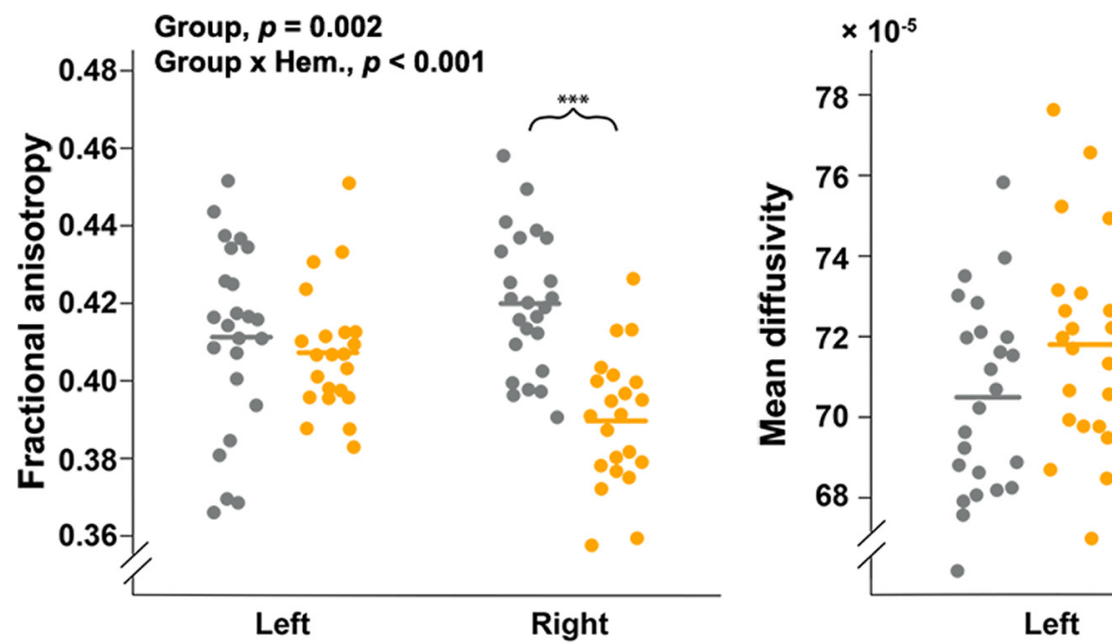

Group, $p=0.036$

Group x Hem., n.s.
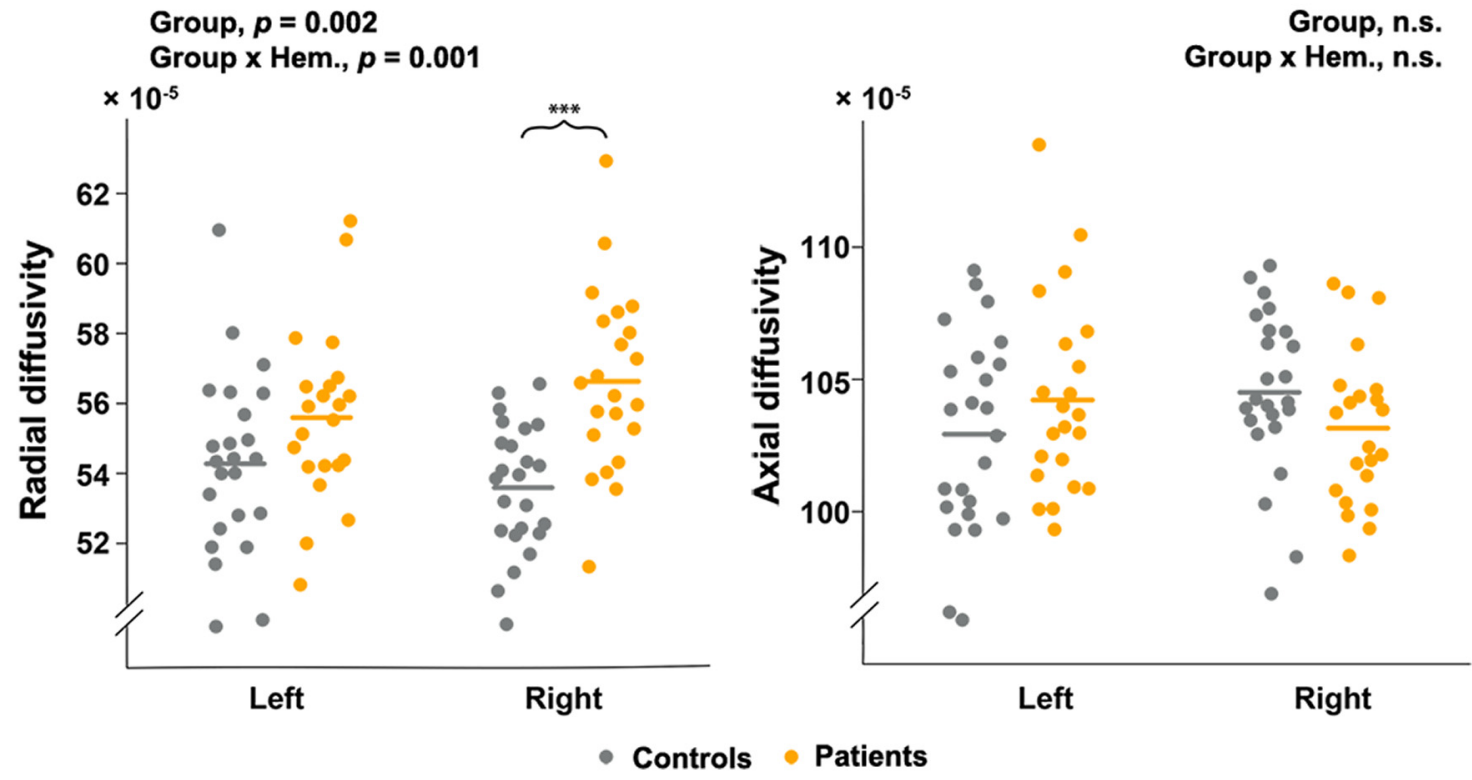

Figure 5. Group differences in the MD-FEF tract microstructural integrity by hemisphere. Top left, Fractional anisotropy. Top right, Mean diffusivity. Bottom left, Radial diffusivity. Bottom right, Axial diffusivity. Higher microstructural integrity (and hence more efficient connectivity) is indicated by higher fractional anisotropy values. Higher diffusivity (and hence lower microstructural integrity in general or along certain directions) is indicated by higher mean diffusivity, radial diffusivity, and axial diffusivity values. Brackets indicate differences between patients and control participants (Figure 5-1, available at https://doi.org/10.1523/JNEUROSCI.1473-18.2019.55-1, ANOVA results in the smaller subsample with behavioral data). Hem, Hemisphere. ${ }^{* * *} p<0.001$.

Table 2. Mean microstructural integrity measures (fractional anisotropy, mean diffusivity, radial diffusivity, axial diffusivity) in the MD-FEF tract by hemisphere (see Table 2-1, available at https://doi.org/10.1523/JNEUROSCl.1473-18.2019.t2-1, for results from the smaller subsample with both DTI data and usable data on the blanking task)

\begin{tabular}{lll}
\hline & $\begin{array}{l}\text { Healthy control } \\
\text { participants } \\
(n=24)\end{array}$ & $\begin{array}{l}\text { Patients with } \\
\text { schizophrenia } \\
(n=22)\end{array}$ \\
\hline $\begin{array}{l}\text { Fractional anisotropy } \\
\quad \text { Left }\end{array}$ & $0.41(0.02)$ & $0.41(0.02)$ \\
$\quad$ Right & $0.42(0.02)$ & $0.39(0.02)$ \\
$\begin{array}{l}\text { Mean diffusivity } \\
\text { Left }\end{array}$ & $7.0 \times 10^{-4}\left(2.40 \times 10^{-5}\right)$ & $7.18 \times 10^{-4}\left(2.65 \times 10^{-5}\right)$ \\
$\quad$ Right & $7.06 \times 10^{-4}\left(1.84 \times 10^{-5}\right)$ & $7.21 \times 10^{-4}\left(2.51 \times 10^{-5}\right)$ \\
$\begin{array}{l}\text { Radial diffusivity } \\
\text { Left }\end{array}$ & $5.43 \times 10^{-4}\left(2.57 \times 10^{-5}\right)$ & $5.56 \times 10^{-4}\left(2.46 \times 10^{-5}\right)$ \\
$\quad$ Right & $5.36 \times 10^{-4}\left(1.82 \times 10^{-5}\right)$ & $5.66 \times 10^{-4}\left(2.59 \times 10^{-5}\right)$ \\
Axial diffusivity & $1.03 \times 10^{-3}\left(3.72 \times 10^{-5}\right)$ & $1.04 \times 10^{-3}\left(3.70 \times 10^{-5}\right)$ \\
$\quad$ Left & $1.05 \times 10^{-3}\left(3.12 \times 10^{-5}\right)$ & $1.03 \times 10^{-3}\left(2.92 \times 10^{-5}\right)$ \\
$\quad$ Right & & \\
\hline Values are reported as the man $(\mathrm{SD})$. &
\end{tabular}

$\eta^{2}=0.20$; mean diffusivity: $F_{(1,44)}=4.69, p=0.036$, partial $\eta^{2}=$ 0.10 ; radial diffusivity: $F_{(1,44)}=10.88, p=0.002$, partial $\eta^{2}=$ $0.20)$. There was no group difference in tract axial diffusivity $\left(F_{(1,44)}=0.001, p=0.98\right.$, partial $\left.\eta^{2}=0.00\right)$. For fractional anisotropy and radial diffusivity, there was an additional group $\times$ hemisphere interaction effect (fractional anisotropy: $F_{(1,44)}=$ 31.98, $p<0.001$, partial $\eta^{2}=0.42$; radial diffusivity: $F_{(1,44)}=$ 13.16, $p=0.001$, partial $\eta^{2}=0.23$ ). Follow-up independent $t$ tests indicated that fractional anisotropy and radial diffusivity differed significantly between groups only in the right hemisphere (fractional anisotropy: $t_{(44)}=5.86, p<0.001$; radial diffusivity: $\left.t_{(44)}=4.63, p<0.001\right)$. This group $\times$ hemisphere interaction effect was not significant for mean diffusivity $\left(F_{(1,44)}=0.42, p=0.52\right.$, partial $\left.\eta^{2}=0.01\right)$. Finally, there was no significant main effect of hemisphere for any of the measures $\left(0.061<p<0.46,0.01<\right.$ partial $\left.\eta^{2}<0.08\right)$. There were no correlations between chlorpromazine equivalent dose and any of the microstructural integrity measures $\left(-0.25<r_{s}<0.19\right.$, $0.30<p<0.98)$. In the subsample of participants with data 


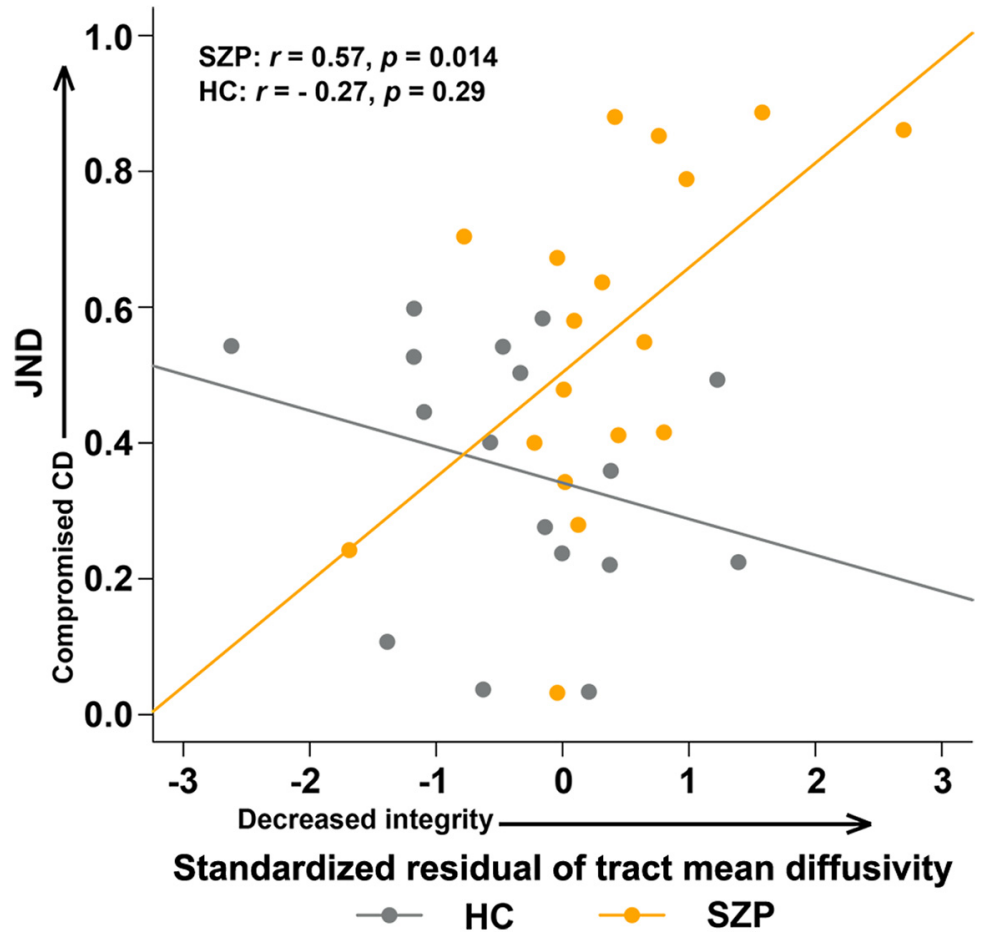

Figure 6. Scatterplot of JND against MD-FEF tract-specific mean diffusivity across hemispheres. These standardized values are 0 when equal to the whole-brain mean diffusivity average, higher for greater diffusivity (i.e., lower microstructural integrity), and lower for less diffusivity (i.e., better microstructural integrity). Smaller JND values are consistent with more intact oculomotor CD signals that inform perception in the blanking task. Compromised oculomotor CD signals correlated with lower white matter microstructure in patients only (Figure 6-1, available at https://doi.org/10.1523/JNEUROSCI.1473-18.2019.f6-1, complete correlation results between standardized residuals of microstructural integrity measures and behavioral CD measures and symptom scores). HC, Healthy control participants; SZP, patients with schizophrenia.

from the blanking task, there was still a significant main effect of group on fractional anisotropy, mean diffusivity, and radial diffusivity, such that patients showed reduced microstructural integrity (Table 2-1, available at https://doi.org/10.1523/ JNEUROSCI.1473-18.2019.t2-1, Fig. 5-1, available at https:// doi.org/10.1523/JNEUROSCI.1473-18.2019.f5-1, complete results from this subsample). There were no correlations between CPZ equivalent dose and any of the microstructural integrity measures in this subsample either $\left(-0.22<r_{s}<0.27,0.27<p<\right.$ $0.73)$.

\section{Previous blanking task findings}

Complete behavioral results can be found in the study by Rösler et al. (2015). Briefly, results were indicative of a reduced influence of CD on perceptual judgements in patients, who showed evidence for relying more on the landing position of the eye to inform perceptual judgments following an eye movement. First, patients exhibited reduced precision of perceptual judgments, as evidenced by a significantly larger JND than in control participants, indicating that their perceptual judgments were less sensitive to the actual target displacement. On a group level, we found a correlation between saccade landing site and perceptual judgements in patients, but not in control participants, such that patients with shorter mean saccade amplitudes made more forward judgements. That is, participants who made shorter saccades on average were more likely to report that the new target jumped forward of the old target location. On the single-subject level, we did not find a significant relationship between saccade landing site and perceptual judgements (i.e., no group difference in loss slope); however, more severe positive symptoms (obtained using the PANSS) were correlated with a more negative loss slope in patients.

\section{Relationship between MD-FEF connectivity and behavioral indices of oculomotor CD}

Because we only found significant group differences in fractional anisotropy, mean diffusivity, and radial diffusivity, we performed Pearson correlation analyses between the standardized residuals of these three measures (to control for variance due to whole-brain white matter integrity differences) and two behavioral measures (loss slope and JND) within each diagnostic group. One patient was excluded from the correlational analyses with JND as this individual's JND was $>3$ SDs away from the group mean. Complete results are reported in Fig. 6-1, available at https://doi.org/10.1523/ JNEUROSCI.1473-18.2019.f6-1. In patients only, we found a significant correlation between the standardized residual of mean diffusivity and JND $(r=0.57, p=$ 0.014; Fig. 6). This is consistent with our prediction: a higher JND, which is indicative of poorer CD signaling, is related to compromised white matter integrity in the MD-FEF pathway in patients. There were no other significant correlations between JND and standardized residuals of diffusivity measures in either group. Finally, no significant correlations were found between the loss slope and the standardized residuals of diffusivity measures in either group.

To examine whether the relationship with behavioral CD indices was unique to the MD-FEF pathway, we also related blanking task performance to structural integrity of a control tract, the corpus callosum. Pearson correlation analyses were conducted between the standardized residuals of the three integrity measures (fractional anisotropy, mean diffusivity, and radial diffusivity) and two behavioral measures (loss slope and JND) within each diagnostic group. We found no significant correlations between either JND or loss slope and standardized residuals of integrity measures in either group $(-0.19<$ all $r$ values $<0.35$, $0.16<$ all $p$ values $<0.91$; Fig. 6-1, available at https://doi.org/ 10.1523/JNEUROSCI.1473-18.2019.f6-1, complete results).

\section{Relationship between MD-FEF connectivity and clinical symptoms}

Last, we conducted Spearman's rank correlation analyses between PANSS subscale scores (positive and negative symptom severity) and the three standardized residuals of diffusivity measures (fractional anisotropy, mean diffusivity, and radial diffusivity) that were altered in patients. We found a significant correlation between standardized mean diffusivity residual and PANSS-positive symptom score $\left(r_{s}=0.43, p=0.047\right)$ and between standardized residual of radial diffusivity in the left tract and PANSS-positive symptom score $\left(r_{s}=0.48, p=0.026\right.$; Fig. 7$)$. No correlations were found between PANSS-negative symptom score and the standardized residuals (Fig. 6-1, available at https:// doi.org/10.1523/JNEUROSCI.1473-18.2019.f6-1, complete re- 

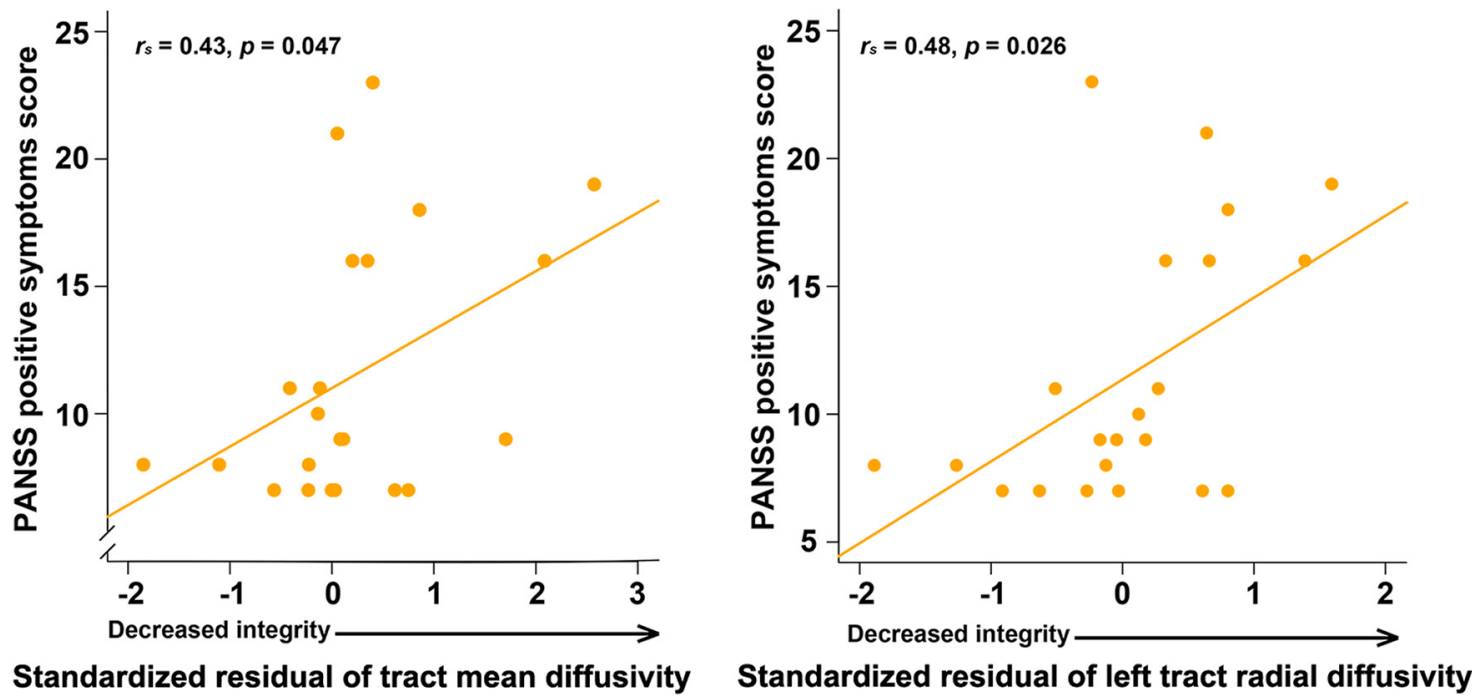

Figure 7. Scatterplots of PANSS-positive symptom scores against MD-FEF tract-specific indices of microstructural integrity. Left, Standardized residuals of mean diffusivity across hemispheres. Right, Standardized residuals of radial diffusivity of the left tract. These standardized values are 0 when equal to the whole-brain mean diffusivity or radial diffusivity average, higher for greater diffusivity (i.e., lower microstructural integrity) and lower for less diffusivity (i.e., better microstructural integrity). Larger PANSS scores indicate more severe symptoms. More severe positive symptoms correlated with more impaired white matter microstructure.

sults). Together, these correlations suggest that more severe positive symptoms in patients, but not negative symptoms, were associated with compromised white matter integrity in the MDFEF pathway.

\section{Discussion}

In this study, we examined microstructural integrity of the MDFEF pathway in schizophrenia patients and the relationship between putative white matter abnormalities and both behavioral indices of oculomotor CD and psychotic symptoms. Using DTI and probabilistic tractography, we found that patients and control participants shared the same spatial location of the tracts connecting MD and FEFs. However, patients had compromised microstructural integrity of these tracts, such that they had lower fractional anisotropy and higher mean diffusivity and radial diffusivity than control participants. This hypoconnectivity was found to further correlate with behavioral indices of reduced oculomotor CD signaling and more severe positive symptoms in patients, indicating a potential disease mechanism that has specific behavioral correlates and symptom implications.

Our finding that schizophrenia patients had reduced MDFEF structural connectivity is consistent with recent findings of white matter alterations in MD-prefrontal pathways in schizophrenia (Giraldo-Chica et al., 2018) and in thalamocortical circuits more generally (for review, see Canu et al., 2015). Similarly, previous studies examining functional connectivity also found reduced thalamofrontal connectivity in patients (Welsh et al., 2010; Woodward et al., 2012; Anticevic et al., 2014) and clinical high-risk populations (Anticevic et al., 2015). Notably, case reports on patients with MD lesions experiencing psychotic-like symptoms such as hallucinations and delusions (Carrera and Bogousslavsky, 2006; Crail-Melendez et al., 2013; Zhou et al., 2015) further support the link between thalamocortical communication and positive symptom formation.

The pattern of group differences across the various diffusivity measures (e.g., axial diffusivity, radial diffusivity, and mean diffusivity) provide further insights into specific structural abnormalities in patients at the neurobiological level. Namely, findings of reduced radial diffusivity paired with no differences in axial diffusivity suggest that decreased fractional anisotropy in the MD-FEF pathway in patients may be driven by myelin and oligodendroglia dysfunction (Davis et al., 2003; Bennett et al., 2010). In addition to demyelination, increased mean diffusivity in patients provides indirect evidence supporting accelerated aging of this pathway, consistent with more general accelerated aging theories of schizophrenia (Islam et al., 2017), as increased mean diffusivity can be a marker of normal aging (Abe et al., 2002). In fact, a recent study (Cropley et al., 2017) found that the rate of white matter decline was $60 \%$ steeper in patients with schizophrenia than in control participants, and such a difference was present throughout the life span.

Somewhat surprisingly, we found that hemisphere moderated group differences in fractional anisotropy and radial diffusivity of the MD-FEF pathway. In the few studies that examined MDcortical functional connectivity, a group $\times$ hemisphere interaction was not modeled, not reported, or not found (Welsh et al., 2010; Woodward et al., 2012; Anticevic et al., 2014). Very few studies have assessed structural connectivity between specific thalamic nuclei and cortical areas in schizophrenia. Consistent with our finding of more prominent group differences in the right hemisphere, a recent structural imaging study (Hamoda et al., 2018) found reduced MD-frontal connectivity in patients in the right hemisphere. Another study (Giraldo-Chica et al., 2018), however, found a more prominent left hemisphere decrease in the prefrontal-MD connectivity. Two key differences distinguished that study from the current one. First, the patient group in the study by Giraldo-Chica et al. (2018) was younger and earlier in the course of illness. Second, and more importantly, their main connectivity analyses used "total connectivity" values (calculated by dividing the number of streamlines reaching a certain cortical area by the number of streamlines reaching all cortical regions from thalamus) rather than indices of microstructural integrity (e.g., fractional anisotropy). In other words, their group comparison revealed the differences in how thalamocortical connectivity was distributed among different cortical regions, rather than the absolute difference in microstructural level within a specific thalamocortical 
pathway. Clearly, more research is needed to address lateralized effects of thalamoprefrontal dysconnectivity, or thalamocortical dysconnectivity in general.

The most novel and important finding of this study is the relationship between reduced MD-FEF structural connectivity and reduced oculomotor CD signal integrity in patients, mirroring findings from primate neurophysiology studies that this pathway plays a crucial role in transmitting CD signals associated with saccades (Sommer and Wurtz, 2002), and similar findings from humans with specific MD lesions (Gaymard et al., 1994; Ostendorf et al., 2010). Based on this rich body of neurophysiology work, one interpretation of the current findings is that reduced connectivity between MD and FEFs leads to a disruption of oculomotor CD signals, resulting in poorer behavioral performance on the blanking task. Presumably, the other consequence of these compromised CD signals were frequent inaccurate predictions about visual inputs, which then led to abnormal perceptual experiences and/or false inferences about the causes of sensory experiences. This could potentially explain the correlation between MD-FEF hypoconnectivity and positive, but not negative, symptom severity. Because variability due to wholebrain white matter diffusivity was partialed out of MD-FEF diffusivity, these relationships among structural connectivity, blanking task performance, and clinical symptoms cannot be explained by global reductions in structural integrity in patients. In addition, these relationships were not observed in the corpus callosum. Despite evidence for relative specificity, there are certainly other thalamocortical pathways involved in CD signal transmission (Sommer and Wurtz, 2004) that were not examined in the current study. Together, the MD-FEF white matter tracts seem to play a key role in transmitting oculomotor CD signals that may also explain some of the symptom manifestation in schizophrenia. This highlights the importance of thalamocortical connectivity as a potential biomarker of and/or a treatment target for schizophrenia (Ramsay and MacDonald, 2018).

Interpretation of the current findings is limited by several factors. First, JND may not be the best indicator of CD signaling in the blanking task because it is also affected by general sources of noise (e.g., in visual input or in motor output). Nevertheless, we observed a relationship between JND and the structural integrity of the MD-FEF pathway in patients. One possible reason that we observed this relationship in patients but not in control participants could be that multiple factors contributed to the variability in JND: increased JND could be caused by a greater reliance on saccade landing site due to dysfunctional CD or nonspecific sources of noise. The former may account for more variability in patients, whereas the latter may account for more variability in control participants. That is, JND may be a more direct measure of $\mathrm{CD}$ in patients than control participants. A second limitation is that we did not include measures specifically assessing agency disturbances, which should be most directly related to CD loss. Nevertheless, altered CD has been posited as a general mechanism underlying hallucinations and delusions (Feinberg and Guazzelli, 1999; Fletcher and Frith, 2009), which were both captured by our measure of positive symptoms. A third limitation is that MD was defined using a probabilistic atlas, as we did not have a means to define it anatomically in each individual. Therefore, the definition of MD might be quite liberal and potentially include ventroanterior parts of the thalamus. However, there is increasing evidence that the thalamus may be considered a hub for relaying CD signals to various cortical projection sites (Sherman, 2016). For instance, there is indirect evidence for a pathway from the ventrolateral nucleus of the thalamus to FEF that conveys CD signals associated with eye velocity during smooth pursuit movements (Tanaka, 2005). Therefore, even if the MD mask includes a small part of other thalamic nuclei, the pathways between these regions and FEF may still play a role in conveying CD signals associated with eye movements and thus be relevant to oculomotor CD dysfunctions in schizophrenia. A fourth potential limitation is that our DTI scans were acquired with 30 different diffusional gradient directions. Although more diffusion directions may have yielded greater accuracy in tractography and tensor-based diffusivity measures, 30 directions has been found to yield reliable results for robust anatomical pathways (Jones, 2004; Heiervang et al., 2006; Testa et al., 2017). Last, we were not able to rule out potential confounding effects of antipsychotic treatment because we used a medicated sample. However, antipsychotic dose did not correlate with any of the behavioral or microstructural measures.

Despite these limitations, we anticipate that findings from the current study will pave the way for more in-depth mechanistic questions on CD and psychosis. Studies using larger sample sizes are necessary to obtain adequate power for regression analyses to test the hypothesis that structural connectivity in the MD-FEF pathway directly mediates the relationship between oculomotor CD signaling abnormalities and positive symptom severity. Second, to establish a causal or directional relationship among white matter integrity, oculomotor CD signals, and psychotic symptoms, future studies should follow clinical high-risk populations longitudinally.

In conclusion, we identified significant decreases in white matter connectivity in patients with schizophrenia relative to control participants in the MD-FEF pathway, which belongs to a key neural circuit transmitting oculomotor CD signals previously established in the animal literature. We demonstrated that hypoconnectivity in the MD-FEF pathway was correlated with impaired oculomotor CD signaling and positive symptoms in patients. Given that the thalamus is poised to play a key role in transmitting CD signals across multiple sensorimotor modalities (Sherman, 2016), these findings have important disease mechanism and treatment implications.

\section{References}

Abe O, Aoki S, Hayashi N, Yamada H, Kunimatsu A, Mori H, Yoshikawa T, Okubo T, Ohtomo K (2002) Normal aging in the central nervous system: quantitative MR diffusion-tensor analysis. Neurobiol Aging 23:433441.

Alexander AL, Lee JE, Lazar M, Field AS (2007) Diffusion tensor imaging of the brain. Neurotherapeutics 4:316-329.

Andersson JL, Skare S (2002) A model-based method for retrospective correction of geometric distortions in diffusion-weighted EPI. Neuroimage 16:177-199.

Andreasen NC, Flaum M, Arndt S (1992) The comprehensive assessment of symptoms and history (CASH). An instrument for assessing diagnosis and psychopathology. Arch Gen Psychiatry 49:615-623.

Anticevic A, Cole MW, Repovs G, Murray JD, Brumbaugh MS, Winkler AM, Savic A, Krystal JH, Pearlson GD, Glahn DC (2014) Characterizing thalamo-cortical disturbances in schizophrenia and bipolar illness. Cereb Cortex 24:3116-3130.

Anticevic A, Haut K, Murray JD, Repovs G, Yang GJ, Diehl C, McEwen SC, Bearden CE, Addington J, Goodyear B, Cadenhead KS, Mirzakhanian H, Cornblatt BA, Olvet D, Mathalon DH, McGlashan TH, Perkins DO, Belger A, Seidman LJ, Tsuang MT, et al. (2015) Association of thalamic dysconnectivity and conversion to psychosis in youth and young adults at elevated clinical risk. JAMA Psychiatry 72:882-891.

Ashburner J, Friston KJ (2005) Unified segmentation. Neuroimage 26:839851 .

Bansal S, Bray LCJ, Schwartz BL, Joiner WM (2018a) Transsaccadic perception deficits in schizophrenia reflect the improper internal monitoring of 
eye movement rather than abnormal sensory processing. Biol Psychiatry Cogn Neurosci Neuroimaging 3:168-177.

Bansal S, Ford JM, Spering M (2018b) The function and failure of sensory predictions. Ann NY Acad Sci. Advance online publication. Retrieved January 9, 2019. doi:10.1111/nyas.13686.

Basser PJ, Pierpaoli C (1996) Microstructural and physiological features of tissues elucidated by quantitative-diffusion-tensor MRI. J Magn Reson B 111:209-219.

Behrens TE, Johansen-Berg H, Woolrich MW, Smith SM, Wheeler-Kingshott CA, Boulby PA, Barker GJ, Sillery EL, Sheehan K, Ciccarelli O, Thompson AJ, Brady JM, Matthews PM (2003a) Non-invasive mapping of connections between human thalamus and cortex using diffusion imaging. Nat Neurosci 6:750-757.

Behrens TE, Woolrich MW, Jenkinson M, Johansen-Berg H, Nunes RG, Clare S, Matthews PM, Brady JM, Smith SM (2003b) Characterization and propagation of uncertainty in diffusion-weighted MR imaging. Magn Reson Med 50:1077-1088.

Behrens TE, Berg HJ, Jbabdi S, Rushworth MF, Woolrich MW (2007) Probabilistic diffusion tractography with multiple fibre orientations: what can we gain? Neuroimage 34:144-155.

Bennett IJ, Madden DJ, Vaidya CJ, Howard DV, Howard JH Jr (2010) Age-related differences in multiple measures of white matter integrity: a diffusion tensor imaging study of healthy aging. Hum Brain Mapp 31:378-390.

Brainard DH (1997) The psychophysics toolbox. Spat Vis 10:433-436.

Canu E, Agosta F, Filippi M (2015) A selective review of structural connectivity abnormalities of schizophrenic patients at different stages of the disease. Schizophr Res 161:19-28.

Carrera E, Bogousslavsky J (2006) The thalamus and behavior: effects of anatomically distinct strokes. Neurology 66:1817-1823.

Cavanagh P, Hunt AR, Afraz A, Rolfs M (2010) Visual stability based on remapping of attention pointers. Trends Cogn Sci 14:147-153.

Cavanaugh J, Berman RA, Joiner WM, Wurtz RH (2016) Saccadic corollary discharge underlies stable visual perception. J Neurosci 36:31-42.

Collins T, Rolfs M, Deubel H, Cavanagh P (2009) Post-saccadic location judgments reveal remapping of saccade targets to non-foveal locations. J Vis 9:29, 1-9.

Cornelissen FW, Peters EM, Palmer J (2002) The eyelink toolbox: eye tracking with MATLAB and the psychophysics toolbox. Behav Res Methods Instrum Comput 34:613-617.

Crail-Melendez D, Atriano-Mendieta C, Carrillo-Meza R, RamirezBermudez J (2013) Schizophrenia-like psychosis associated with right lacunar thalamic infarct. Neurocase 19:22-26.

Cropley VL, Klauser P, Lenroot RK, Bruggemann J, Sundram S, Bousman C, Pereira A, Di Biase MA, Weickert TW, Weickert CS, Pantelis C, Zalesky A (2017) Accelerated gray and white matter deterioration with age in schizophrenia. Am J Psychiatry 174:286-295.

Davis KL, Stewart DG, Friedman JI, Buchsbaum M, Harvey PD, Hof PR, Buxbaum J, Haroutunian V (2003) White matter changes in schizophrenia. Arch Gen Psychiatry 60:443-456.

Deubel H, Schneider WX, Bridgeman B (1996) Postsaccadic target blanking prevents saccadic suppression of image displacement. Vision Res 36:985996.

Feinberg I (1978) Efference copy and corollary discharge: implications for thinking and its disorders. Schizophr Bull 4:636-640.

Feinberg I, Guazzelli M (1999) Schizophrenia—a disorder of the corollary discharge systems that integrate the motor systems of thought with the sensory systems of consciousness. Br J Psychiatry 174:196-204.

Fletcher PC, Frith CD (2009) Perceiving is believing: a Bayesian approach to explaining the positive symptoms of schizophrenia. Nat Rev Neurosci $10: 48-58$.

Gaymard B, Rivaud S, Pierrot-Deseilligny C (1994) Impairment of extraretinal eye position signals after central thalamic lesions in humans. Exp Brain Res 102:1-9.

Genetic Risk and Outcome in Psychosis (GROUP) Investigators (2011) Evidence that familial liability for psychosis is expressed as differential sensitivity to cannabis: an analysis of patient-sibling and sibling-control pairs. Arch Gen Psychiatry 68:138-147.

Giraldo-Chica M, Rogers BP, Damon SM, Landman BA, Woodward ND (2018) Prefrontal-thalamic anatomical connectivity and executive cognitive function in schizophrenia. Biol Psychiatry 83:509-517.

Hammers A, Allom R, Koepp MJ, Free SL, Myers R, Lemieux L, Mitchell TN, Brooks DJ, Duncan JS (2003) Three-dimensional maximum probability atlas of the human brain, with particular reference to the temporal lobe. Hum Brain Mapp 19:224-247.

Hamoda HM, Makhlouf AT, Fitzsimmons J, Rathi Y, Makris N, MesholamGately RI, Wojcik JD, Goldstein J, McCarley RW, Seidman LJ, Kubicki M, Shenton ME (2018) Abnormalities in thalamo-cortical connections in patients with first-episode schizophrenia: a two-tensor tractography study. Brain Imaging Behav. Advance online publication. Retrieved January 9, 2019. doi:10.1007/s11682-018-9862-8.

Heiervang E, Behrens TE, Mackay CE, Robson MD, Johansen-Berg H (2006) Between session reproducibility and between subject variability of diffusion MR and tractography measures. Neuroimage 33:867-877.

Islam F, Mulsant BH, Voineskos AN, Rajji TK (2017) Brain-derived neurotrophic factor expression in individuals with schizophrenia and healthy aging: testing the accelerated aging hypothesis of schizophrenia. Curr Psychiatry Rep 19:36.

Johansen-Berg H, Behrens TE, Sillery E, Ciccarelli O, Thompson AJ, Smith SM, Matthews PM (2005) Functional-anatomical validation and individual variation of diffusion tractography-based segmentation of the human thalamus. Cereb Cortex 15:31-39.

Joiner WM, Cavanaugh J, FitzGibbon EJ, Wurtz RH (2013) Corollary discharge contributes to perceived eye location in monkeys. J Neurophysiol 110:2402-2413.

Jones DK (2004) The effect of gradient sampling schemes on measures derived from diffusion tensor MRI: a Monte Carlo study. Magn Reson Med 51:807-815.

Kay SR, Fiszbein A, Opler LA (1987) The positive and negative syndrome scale (PANSS) for schizophrenia. Schizophr Bull 13:261-276.

Kelly S, Jahanshad N, Zalesky A, Kochunov P, Agartz I, Alloza C, Andreassen OA, Arango C, Banaj N, Bouix S, Bousman CA, Brouwer RM, Bruggemann J, Bustillo J, Cahn W, Calhoun V, Cannon D, Carr V, Catts S, Chen J, et al. (2018) Widespread white matter microstructural differences in schizophrenia across 4322 individuals: results from the ENIGMA schizophrenia DTI working group. Mol Psychiatry 23:1261-1269.

Landman BA, Bogovic JA, Wan H, El Zahraa ElShahaby F, Bazin PL, Prince JL (2012) Resolution of crossing fibers with constrained compressed sensing using diffusion tensor MRI. Neuroimage 59:2175-2186.

Marenco S, Stein JL, Savostyanova AA, Sambataro F, Tan HY, Goldman AL, Verchinski BA, Barnett AS, Dickinson D, Apud JA, Callicott JH, MeyerLindenberg A, Weinberger DR (2012) Investigation of anatomical thalamo-cortical connectivity and fMRI activation in schizophrenia. Neuropsychopharmacology 37:499-507.

Mori S, van Zijl PC (2002) Fiber tracking: principles and strategies-a technical review. NMR Biomed 15:468-480.

Mori S, Zhang J (2006) Principles of diffusion tensor imaging and its applications to basic neuroscience research. Neuron 51:527-539.

Mulder J, Bouma JM, Schmand B (2012) Nederlandse leestest voor volwassenen. In: Handboek neuropsychologische diagnostiek (Bouma JM, Mulder J, Lindeboom J, Schmand B, eds), pp 127-138. Amsterdam: Pearson Assessment and Information.

Neggers SF, van Diepen RM, Zandbelt BB, Vink M, Mandl RC, Gutteling TP (2012) A functional and structural investigation of the human frontobasal volitional saccade network. PLoS One 7:e29517.

Ostendorf F, Liebermann D, Ploner CJ (2010) Human thalamus contributes to perceptual stability across eye movements. Proc Natl Acad Sci U S A 107:1229-1234.

Pergola G, Selvaggi P, Trizio S, Bertolino A, Blasi G (2015) The role of the thalamus in schizophrenia from a neuroimaging perspective. Neurosci Biobehav Rev 54:57-75.

Ramsay IS, MacDonald AW 3rd (2018) The ups and downs of thalamocortical connectivity in schizophrenia. Biol Psychiatry 83:473-474.

Rösler L, Rolfs M, van der Stigchel S, Neggers SF, Cahn W, Kahn RS, Thakkar KN (2015) Failure to use corollary discharge to remap visual target locations is associated with psychotic symptom severity in schizophrenia. J Neurophysiol 114:1129-1136.

Sherman SM (2016) Thalamus plays a central role in ongoing cortical functioning. Nat Neurosci 19:533-541.

Smith SM, Jenkinson M, Woolrich MW, Beckmann CF, Behrens TE, Johansen-Berg H, Bannister PR, De Luca M, Drobnjak I, Flitney DE, Niazy RK, Saunders J, Vickers J, Zhang Y, De Stefano N, Brady JM, Matthews PM (2004) Advances in functional and structural MR image analysis and implementation as FSL. Neuroimage 23:S208-S219.

Snook L, Plewes C, Beaulieu C (2007) Voxel based versus region of interest 
analysis in diffusion tensor imaging of neurodevelopment. Neuroimage 34:243-252.

Sommer MA, Wurtz RH (2002) A pathway in primate brain for internal monitoring of movements. Science 296:1480-1482.

Sommer MA, Wurtz RH (2004) What the brain stem tells the frontal cortex. II. role of the SC-MD-FEF pathway in corollary discharge. J Neurophysiol 91:1403-1423.

Sommer MA, Wurtz RH (2008) Brain circuits for the internal monitoring of movements. Annu Rev Neurosci 31:317-338.

Song SK, Sun SW, Ramsbottom MJ, Chang C, Russell J, Cross AH (2002) Dysmyelination revealed through MRI as increased radial (but unchanged axial) diffusion of water. Neuroimage 17:1429-1436.

Tanaka M (2005) Involvement of the Central Thalamus in the Control of Smooth Pursuit Eye Movements. J Neurosci 25:5866-5876.

Testa C, Evangelisti S, Popeo M, Zanigni S, Gramegna LL, Fantazzini P, Tonon C, Manners DN, Lodi R (2017) The effect of diffusion gradient direction number on corticospinal tractography in the human brain: an along-tract analysis. MAGMA 30:265-280.
Thakkar KN, van den Heiligenberg FM, Kahn RS, Neggers SF (2014) Frontal-subcortical circuits involved in reactive control and monitoring of gaze. J Neurosci 34:8918-8929.

Thakkar KN, Diwadkar VA, Rolfs M (2017) Oculomotor prediction: a window into the psychotic mind. Trends Cogn Sci 21:344-356.

Welsh RC, Chen AC, Taylor SF (2010) Low-frequency BOLD fluctuations demonstrate altered thalamocortical connectivity in schizophrenia. Schizophr Bull 36:713-722.

Wing JK, Babor T, Brugha T, Burke J, Cooper JE, Giel R, Jablenski A, Regier D, Sartorius N (1990) SCAN: schedules for clinical assessment in neuropsychiatry. Arch Gen Psychiatry 47:589-593.

Woods SW (2003) Chlorpromazine equivalent doses for the newer atypical antipsychotics. J Clin Psychiatry 64:663-667.

Woodward ND, Karbasforoushan H, Heckers S (2012) Thalamocortical dysconnectivity in schizophrenia. Am J Psychiatry 169:1092-1099.

Zhou Y, Fox D, Anand A, Elhaj A, Kapoor A, Najibi F, Kim H, Weir R, Jayam-Trouth A (2015) Artery of percheron infarction as an unusual cause of Korsakoff's syndrome. Case Rep Neurol Med 2015:927809. 